\title{
Tracheal Structure Characterization using Geometric and Appearance Models for Efficient Assessment of Stenosis in Videobronchoscopy
}

\author{
Carles Sánchez \\ Computer Science Department \& Computer Vision Center, Edifici Q/O Campus UAB, Cerdanyola del Valles, Barcelona, Spain \\ Advisor/s: F. Javier Sánchez, Debora Gil and Jorge Bernal \\ Date and location of PhD thesis defense: 16 December 2014, Autonomous University of Barcelona
}

Received 26th February 2015; accepted 14th May 2015

\begin{abstract}
Recent advances in endoscopic devices have increased their use for minimal invasive diagnostic and intervention procedures. Among all endoscopic modalities, bronchoscopy is one of the most frequent with around 261 millions of procedures per year. Although the use of bronchoscopy is spread among clinical facilities it presents some drawbacks, being the visual inspection for the assessment of anatomical measurements the most prevalent of them. In particular, inaccuracies in the estimation of the degree of stenosis (the percentage of obstructed airway) decreases its diagnostic yield and might lead to erroneous treatments. An objective computation of tracheal stenosis in bronchoscopy videos would constitute a breakthrough for this non-invasive technique and a reduction in treatment cost.

This thesis settles the first steps towards on-line reliable extraction of anatomical information from videobronchoscopy for computation of objective measures. In particular, we focus on the computation of the degree of stenosis, which is obtained by comparing the area delimited by a healthy tracheal ring and the stenosed lumen (see fig. 1 showing the different steps). In this sense, we have to consider that reliable extraction of airway structures in interventional videobronchoscopy is a challenging task. This is mainly due to the large variety of acquisition conditions (positions and illumination), devices (different digitalizations) and in videos acquired at the operating room the unpredicted presence of surgical devices (such as probe ends). This thesis contributes to on-line stenosis assessment in several ways. We propose a parametric strategy for the extraction of lumen and tracheal rings regions based on the characterization of their geometry and appearance that guide a deformable model. The geometric and appearance characterization is based on a physical model describing the way bronchoscopy images are obtained and includes local and global descriptions. In order to ensure a systematic applicability we present a statistical framework to select the optimal parameters of our method. Experiments perform on the first public annotated database, show that the performance of our method is comparable to the one provided by clinicians and its computation time allows for a on-line implementation in the operating room.
\end{abstract}

Correspondence to: <csanchez@cvc.uab.cat>

Recommended for acceptance by Jorge Bernal

DOI http://dx.doi.org/10.5565/rev/elcvia. 720

ELCVIA ISSN:1577-5097

Published by Computer Vision Center / Universitat Autònoma de Barcelona, Barcelona, Spain 


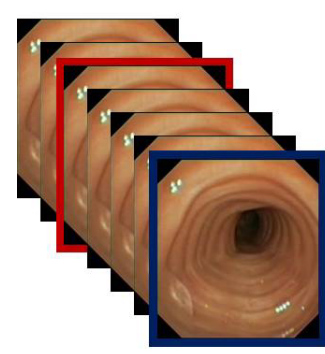

Video exploration

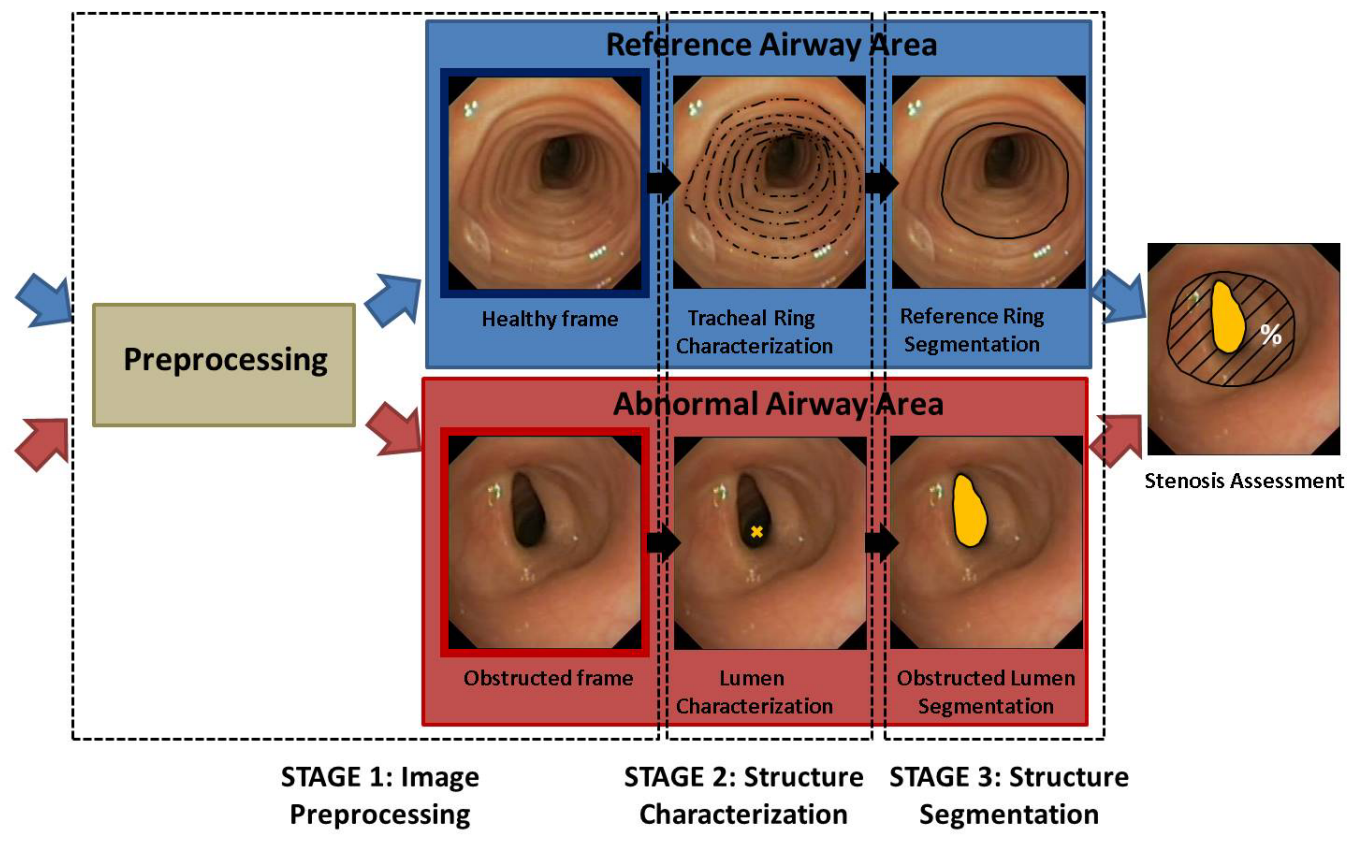

Figure 1: Stenosis assessment workflow.

\section{References}

[1] Carles Sánchez, Jorge Bernal, F. Javier Sánchez, Marta Diez-Ferrer, Antoni Rosell \& Debora Gil. (2015) "Towards On-line Quantification of Tracheal Stenosis from Videobronchoscopy". International Journal of Computer Assisted Radiology and Surgery, IJCARS, (Vol.10, pp. 935-945) Springer.

[2] Carles Sánchez, Jorge Bernal, Debora Gil, \& F. Javier Sánchez. (2014). "On-line lumen centre detection in gastrointestinal and respiratory endoscopy". In Klaus Miguel Angel and Drechsler Stefan and Gonzlez Ballester Raj and Wesarg Cristina and Shekhar M. G. and O. L. Marius and Linguraru (Ed.), Clinical Image-Based Procedures. Translational Research in Medical Imaging (CLIP-MICCAI) (Vol. 8361, pp. 3138). LNCS. Springer International Publishing.

[3] Jorge Bernal, Debora Gil, Carles Sánchez, \& F. Javier Sánchez. (2014). ”Discarding Non Informative Regions for Efficient Colonoscopy Image Analysis". In 1st MICCAI Workshop on Computer-Assisted and Robotic Endoscopy.

[4] Carles Sánchez, Debora Gil, Antoni Rosell, Albert Andaluz, \& F. Javier Sánchez. (2013). "Segmentation of Tracheal Rings in Videobronchoscopy combining Geometry and Appearance". In Sebastiano Battiato and Jos Braz (Ed.), Proceedings of VISAPP 2013 (Vol. 1, pp. 153-161). LNCS. Portugal: SciTePress.

[5] Carles Sánchez, F. Javier Sánchez, Antoni Rosell, \& Debora Gil. (2012). "An illumination model of the trachea appearance in videobronchoscopy images". In Image Analysis and Recognition (Vol. 7325, pp. 313-320). LNCS. Springer Berlin Heidelberg. 